\title{
Cardiovascular Outcomes with Once-Weekly GLP-1 RAs: Clinical and Economic Implications
}

\author{
Michael Heile, MD, FAAFP; Kathleen Wyne, MD, PhD, FACE, FNLA; Liana K. Billings, MD, MMSc; \\ Anthony Cannon, MD, FACE; Yehuda Handelsman, MD, FACP, FNLA, FASPC, MACE; \\ and Michael Shannon, MD
}

\begin{abstract}
Type 2 diabetes mellitus (T2DM) is associated with an increased risk of atherosclerotic cardiovascular (ASCVD) disease, which is the largest contributor to the economic burden of diabetes. Minimization of disease morbidity through comprehensive management of ASCVD risk factors, including but not limited to hyperglycemia, is a key goal of T2DM therapy. Emerging evidence with some glucagon-like peptide-1 receptor agonists (GLP-1 RAs) points to beneficial effects across a range of atherosclerotic risk factors and possible improvement of some cardiovascular outcomes independent of these effects. Given these benefits, there has been substantial interest in evaluating the cardiovascular safety of GLP-1 RAs as well as their potential to reduce the risk of major adverse cardiac events (MACE). Following the superior clinical outcome with the once-daily GLP-1 RA liraglutide (Liraglutide Effect and Action in Diabetes: Evaluation of cardiovascular outcome Results [LEADER]), this review examines and summarizes the effects of once-weekly GLP-1 RAs, including exenatide extended release (ER), dulaglutide, and semaglutide, on reducing cardiovascular events in patients with T2DM. A phase 3 cardiovascular outcomes trial (EXSCEL) of exenatide ER found no significant difference between exenatide ER and placebo in reducing MACE in patients with T2DM. In a phase 3 premarketing trial in T2DM patients at high risk of cardiovascular disease (SUSTAIN-6), semaglutide significantly reduced the risks of MACE and non-fatal stroke compared with placebo. A phase 3 study (REWIND) is underway to evaluate the effects of dulaglutide on MACE. Considering the substantial costs of cardiovascular disease in patients with T2DM, it will be of interest to assess the impact of treatment with once-weekly GLP-1 RAs on cardiovascular disease-related costs among patients with T2DM.
\end{abstract}

J Manag Care Spec Pharm. 2018;24(9-a):S42-S52

Copyright @ 2018, Academy of Managed Care Pharmacy. All rights reserved.

$\mathrm{T}$ ype 2 diabetes mellitus (T2DM) is associated with an increased risk of multiple comorbidities due to insulin resistance, hyperglycemia-induced abnormalities, oxidative stress, and inflammation throughout the vascular system. ${ }^{1}$ Atherosclerotic cardiovascular disease (ASCVD) encompasses cardiac-related events such as acute coronary syndromes, stable or unstable angina, transient ischemic attack, history of myocardial infarction (MI), coronary or arterial revascularization, stroke, and peripheral arterial disease. ${ }^{2}$ Both ASCVD and related risk factors are common among patients with diabetes. ${ }^{1}$ There is a 2- to 4 -fold increased risk of ASCVD development in people with T2DM. ${ }^{1}$ In addition, ASCVD is the primary cause of death in patients with T2DM and has been established as the largest contributor to the direct and indirect costs of diabetes. ${ }^{2}$

Several studies have examined whether intensive blood glucose control (i.e., targeting glycated hemoglobin [Alc] levels as low as 6.0\%-6.5\%) can reduce CV risk, but collective findings have been inconclusive. The United Kingdom Prospective Diabetes Study (UKPDS) observed a trend toward reduced cardiovascular risk (MI) with intensive glycemic control over 10 years, but it was not statistically significant ${ }^{3}$; however, post-trial monitoring of the UKPDS for an additional 10 years demonstrated significant reductions in the risks of MI among patients with new-onset diabetes initially assigned to intensive glycemic control (15\% reduction; $P=0.01$ ) and all-cause mortality $(P=0.007) .{ }^{4}$ Additional studies with median follow-up periods of 3.4-5.6 years, including the Action in Diabetes and Vascular Disease (ADVANCE) study, ${ }^{5}$ the Action to Control Cardiovascular Risk in Diabetes (ACCORD) study, ${ }^{6}$ and the Veterans Affairs Diabetes Trial (VADT), ${ }^{7}$ did not observe a significant benefit of intensive glycemic control on primary cardiovascular endpoints; further, the ACCORD study observed increased all-cause mortality in the intensive therapy arm. In the Steno-2 study (mean follow-up, 7.8 years), a significant reduction in cardiovascular risk in patients with T2DM and microalbuminuria was achieved with an intensive multifactorial strategy, but patients remain at risk. ${ }^{8}$

A meta-analysis of randomized rosiglitazone trials with more than 24 weeks of drug exposure suggested an increased risk of MI and death from ASCVD causes associated with rosiglitazone treatment. ${ }^{9}$ Subsequently, based on the RECORD trial, ${ }^{10}$ the U.S. Food and Drug Administration (FDA) recognized that rosiglitazone does not increase the risk of overall cardiovascular morbidity or mortality relative to other antidiabetic drugs. However, by that time, the FDA had implemented new antidiabetes drug cardiovascular safety guidelines. The FDA "Guidance for Industry" for new diabetes drugs, enacted in 2008, requires evidence of a lack of substantial increase in cardiovascular risk ${ }^{11}$ either through meta-analysis of phase 2-3 trials or through clinical trials showing noninferiority versus placebo for cardiovascular outcomes. Given the mixed effect of glycemic control and residual ASCVD risk despite multifactorial therapy and data raising suggestions of increased ASCVD risk with certain antidiabetic agents, ${ }^{9}$ long-term cardiovascular outcome trials for antidiabetes medications are essential to examine their effect on ASCVD risk. An ideal goal of T2DM therapy would be to improve a wide range of health outcomes and minimize disease morbidity through effective and costeffective comprehensive management of blood glucose as well as all ASCVD risk factors. ${ }^{2}$ As described below, glucagon-like peptide-1 receptor agonists (GLP-1 RAs) exert beneficial effects across a range of ASCVD risk factors. 


\section{TABLE 1 Impact of Once-Weekly GLP-1 RAs on Cardiovascular Risk Factors: Change from Baseline} (Ranges Reported in Clinical Studies)

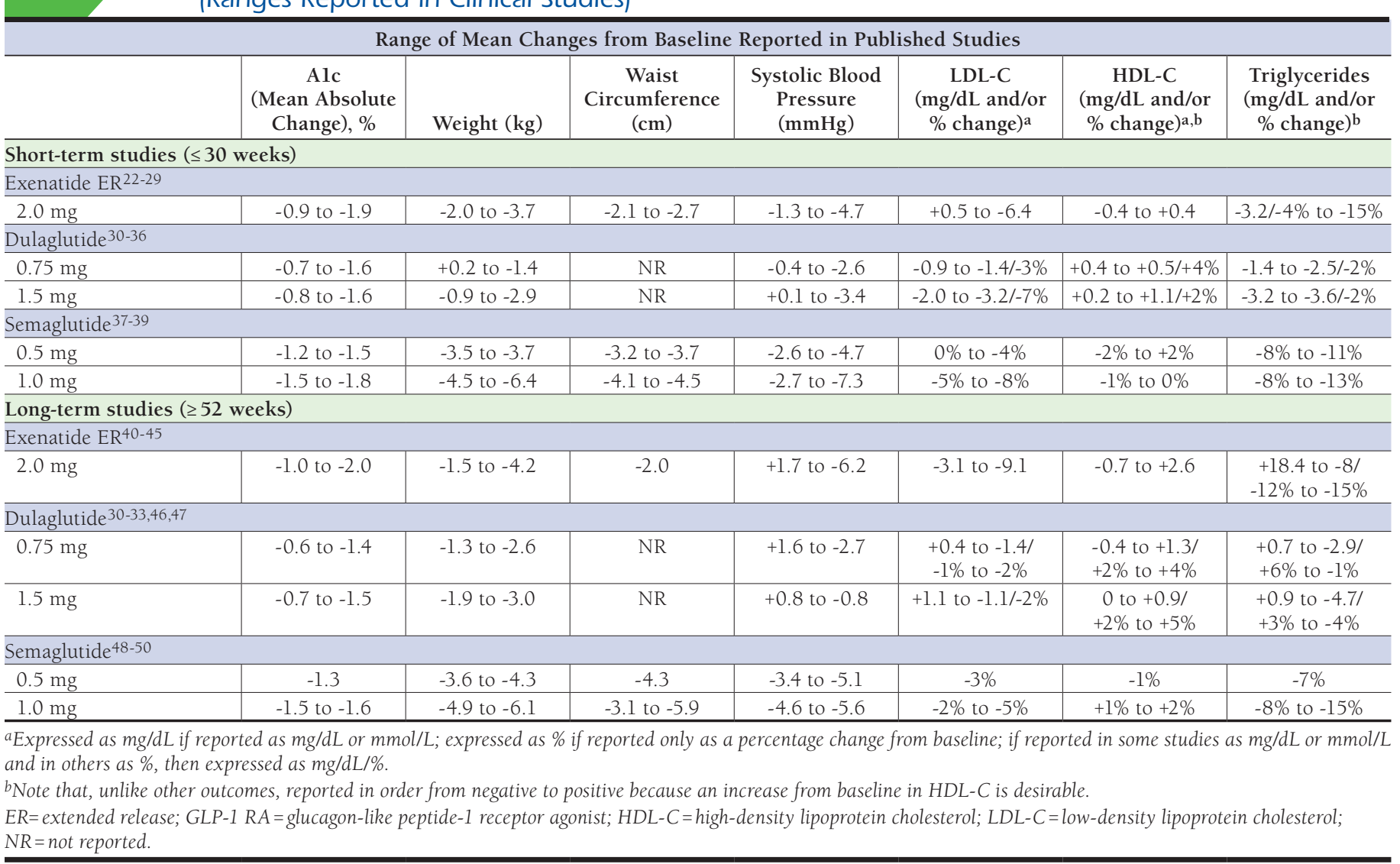

\section{Glucagon-Like Peptide-1 Receptor Agonists}

GLP-1 RAs activate the GLP-1 receptor on pancreatic beta cells to stimulate glucose-dependent insulin secretion. They also slow gastric emptying and increase satiety, which leads to reduced food intake., ${ }^{2,12,13}$ Indirect effects on glucagon secretion have also been described, resulting in normalization of the abnormally elevated glucagon levels seen in T2DM.,12-14 The first approved GLP-1 RA was exenatide (Byetta; AstraZeneca Pharmaceuticals, Wilmington, DE), ${ }^{15}$ which is administered twice daily via subcutaneous (SC) injection. Subsequently approved GLP-1 RAs with once-daily SC dosing include liraglutide (Victoza, Novo Nordisk, Plainsboro, NJ), ${ }^{16}$ and, more recently, the short-acting lixisenatide (Adlyxin; SanofiAventis U.S., Bridgewater, NJ). ${ }^{17}$ Other agents have prolonged action and can be administered once weekly, including exenatide extended-release (ER; Bydureon; AstraZeneca Pharmaceuticals, Wilmington, DE) ${ }^{18}$ dulaglutide (Trulicity; Eli Lilly and Company, Indianapolis, IN), ${ }^{19}$ and semaglutide (Ozempic; Novo Nordisk, Plainsboro, NJ). ${ }^{20}$

To varying degrees, class advantages of GLP-1 RAs include effective lowering of Alc levels, weight loss, and a low risk of hypoglycemia. ${ }^{2,21}$ As a class, the GLP-1 RAs are unique in that they have beneficial effects across a wide range of individual atherosclerotic risk factors, including hyperglycemia, overweight/central obesity, hypertension, and dyslipidemia. Table 1 describes the ranges of effects of once-weekly GLP-1 RAs on these factors as observed in phase 3 clinical studies. Although Table 1 indicates differences between individual once-weekly GLP-1 RAs with regard to their effects on various atherosclerotic risk factors, it should be considered that differences in patient selection criteria and background medication may have contributed to observed disparities.

Given the beneficial effects of GLP-1 RAs in several organ systems that affect cardiovascular health and the localization of GLP-1 receptors in cardiac and vascular tissues, researchers have suspected that GLP-1 RAs might have the potential to improve cardiovascular outcomes (Figure 1). ${ }^{51}$ Accumulating evidence suggests that GLP-1 RAs may favorably affect cardiovascular risk through direct actions on the myocardium and blood vessels. ${ }^{51-53}$ Animal studies have associated GLP-1 RAs with inflammatory response inhibition, increased coronary flow, and additional benefits for myocardial function. ${ }^{54}$ 


\section{FIGURE 1 Potential Indirect Cardiovascular Effects of GLP-1 RAs ${ }^{51}$}

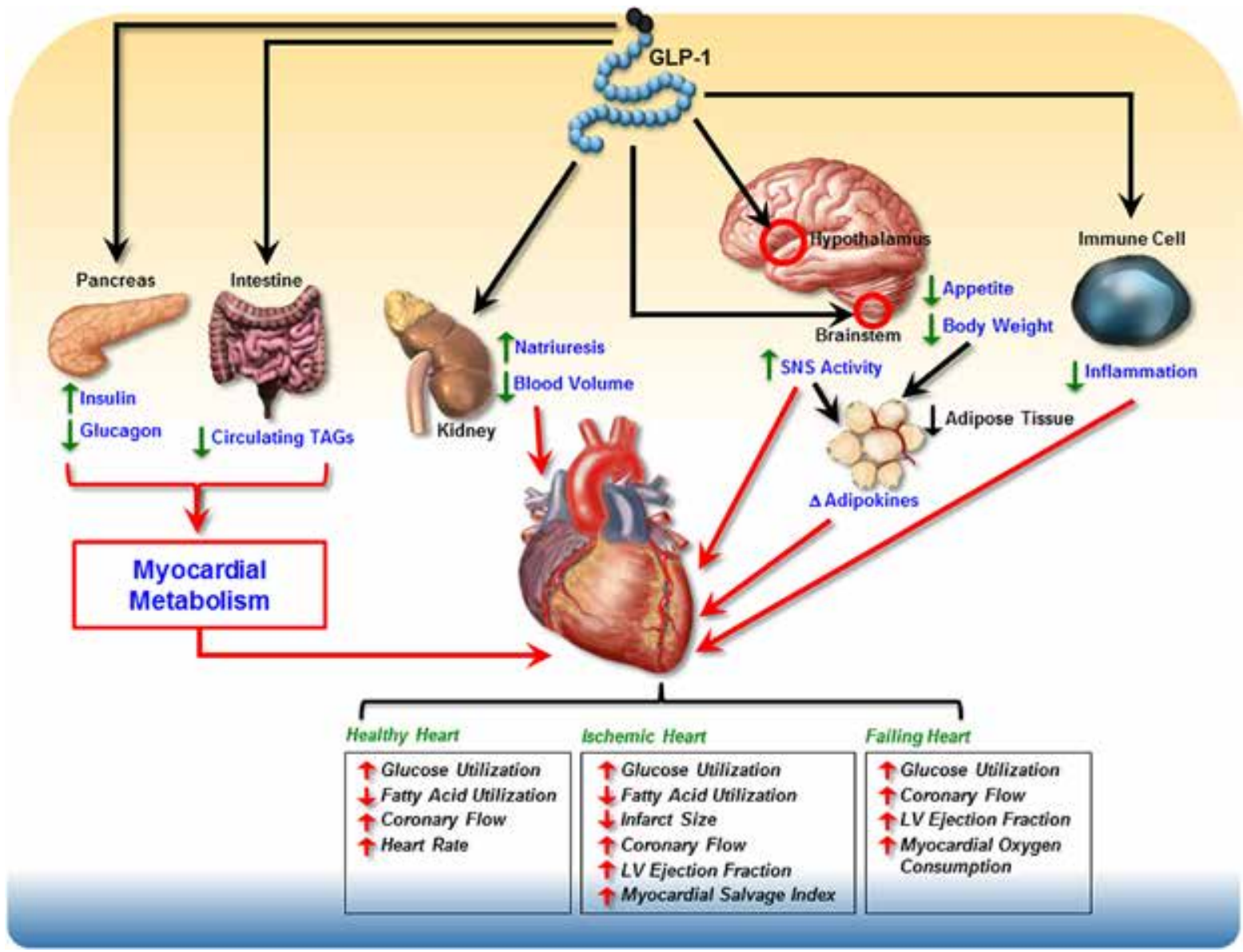

From Ussher JR, Drucker DJ. Cardiovascular actions of incretin-based therapies. Circ Res. 2014;114(11):1788-803 (https://www.ahajournals.org/doi/10.1161/CIRCRESAHA. 114.301958?url_ver=Z39.88-2003Erfr_id=ori:rid:crossref.orgErfr_dat=cr_pub\%3dpubmed). Copyright ( 2014 . Reprinted with permission.

Note: Direct actions of GLP-1 on islets result in elevated insulin and reduced glucagon levels, whereas GLP-1 action in the intestine has been associated with reductions in circulating lipids. These metabolic alterations decrease fatty acid delivery and enhance glucose uptake in the heart and may explain the increased glucose/decreased fatty acid utilization and enhanced LV function observed in the myocardium after systemic GLP-1 R activation. Direct actions on immune cells to decrease inflammation may also indirectly improve myocardial metabolism and subsequent contractile function. Furthermore, direct actions on the SNS may contribute to GLP-1-induced alterations in heart rate, whereas the reduction in appetite after hypothalamic GLP-1 R activation results in reductions in adiposity and body weight, which, combined with the direct and indirect effects on the kidney to decrease blood volume (through increased natriuresis), likely contribute to the overall improvement in LV function after systemic GLP-1 $R$ activation.

GLP-1 = glucagon-like peptide-1 GLP-1 R=glucagon-like peptide-1 receptor; GLP-1 RA=glucagon-like peptide-1 receptor agonist; $L V=l$ eft ventricular; SNS =sympathetic nervous system; $T A G=$ triacylglycerol.

Relevant research in human populations requires large patient numbers and long-term studies, but such data are now starting to come to fruition, including some landmark trials with the once-daily GLP-1 RAs liraglutide and lixisenatide. The long-term cardiovascular benefits of liraglutide were illustrated in the Liraglutide Effect and Action in Diabetes: Evaluation of cardiovascular outcome Results (LEADER) study, an international, double-blind trial that randomized 9,340 patients with T2DM at high risk of cardiovascular disease to liraglutide or placebo. ${ }^{52}$ This study showed that liraglutide significantly
$(P=0.01)$ reduced the risk of a composite major adverse cardiac event (MACE) outcome of death from cardiovascular causes, nonfatal MI, or nonfatal stroke compared with placebo (hazard ratio $[\mathrm{HR}]=0.87$; 95\% confidence interval $[\mathrm{CI}]=0.78-0.97)$ and also significantly reduced the rate of death from cardiovascular causes $(\mathrm{HR}=0.78 ; 95 \% \mathrm{CI}=0.66-0.93 ; P=0.007)$ and the rate of all-cause mortality ( $\mathrm{HR}=0.85 ; 95 \% \mathrm{CI}=0.74-0.97 ; P=0.02$ ).

The Evaluation of Lixisenatide in Acute Coronary Syndrome (ELIXA) trial $(\mathrm{N}=6,068)$ observed no significant differences between lixisenatide and placebo in the occurrence of MACE, 


\section{TABLE 2 Studies Evaluating MACE with Once-Weekly GLP-1 RAs}

Exenatide QW: EXSCEL trial ${ }^{61}$

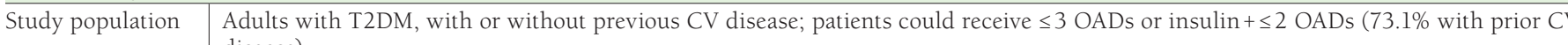
disease)

\begin{tabular}{l|l}
\hline Number/ & Exenatide QW, $\mathrm{n}=7,356$; placebo, $\mathrm{n}=7,396$
\end{tabular}

observation period Median observation period $=3.2$ years

\begin{tabular}{l|l}
\hline CV-related & Primary: Composite outcome: first occurrence of death from CV causes, nonfatal MI, or nonfatal stroke
\end{tabular}

endpoints Secondary: Death from any cause, death from CV causes, and the first occurrence of nonfatal or fatal MI, nonfatal or fatal stroke, hospitalization for acute coronary syndrome, and hospitalization for heart failure in time-to-event analyses

Topline findings $\quad$ Primary: $11.4 \%$ in the exenatide group (3.7 events per 100 person-years) vs. $12.2 \%$ in the placebo group (4.0 events per 100 person-years; $\mathrm{HR}=0.91 ; 95 \% \mathrm{CI}=0.83-1.00)$

Secondary: The rates of the first fatal or nonfatal MI, fatal or nonfatal stroke, and other secondary outcomes were not significantly different between the 2 groups

\begin{tabular}{l|l}
\hline Conclusions & The incidence of MACE was not significantly different between exenatide QW and placebo treatment
\end{tabular}

Dulaglutide: REWIND trial (ongoing) 62

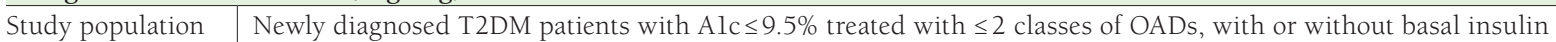

Additional eligibility requirements: 50-54 years of age: previous CV disease; 50-59 years of age: either previous CV disease or evidence of other vascular or renal disease; aged $\geq 60$ years: previous CV disease, other vascular or renal disease, or $\geq 2$ other CV risk factors

\begin{tabular}{l|ll}
\hline Number/ \\
observation period
\end{tabular}

9,901 participants recruited

CV-related $\quad$ Primary: First occurrence of the composite of cardiovascular death or nonfatal MI or nonfatal stroke \begin{tabular}{l|l} 
endpoints & $\begin{array}{l}\text { Secondary: Each component of the primary composite CV outcome, a composite clinical microvascular outcome comprising retinal or } \\
\text { renal disease, hospitalization for unstable angina, heart failure requiring hospitalization or an urgent heart failure visit, and all-cause }\end{array}$
\end{tabular} mortality

\begin{tabular}{l|l}
\hline Topline findings & TBD (expected completion July 2018)
\end{tabular}

\begin{tabular}{l|l}
\hline Conclusions & TBD (expected completion July 2018) \\
\hline
\end{tabular}

Semaglutide: SUSTAIN-6 study 50

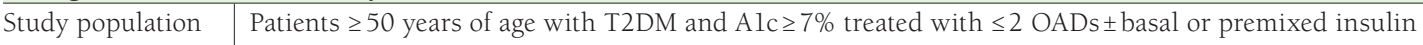

Patients were at high CV risk

Number/ $\quad$ Semaglutide, $\mathrm{n}=1,648$; placebo, $\mathrm{n}=1,649$

observation period Median observation period $=2.1$ years

\begin{tabular}{l|l}
\hline CV-related & Primary: Composite outcome: first occurrence of CV death, nonfatal MI, or nonfatal stroke
\end{tabular}

endpoints $\quad$ Secondary: First occurrence of an expanded composite CV outcome (death from CV causes, nonfatal MI, nonfatal stroke, revascularization [coronary or peripheral], and hospitalization for unstable angina or heart failure), an additional composite outcome (death from all causes, nonfatal MI, or nonfatal stroke), or the individual components of the composite outcome

Topline findings $\quad$ Primary: Semaglutide-treated patients had a significant 26\% lower risk of the primary composite outcome; $6.6 \%$ in the semaglutide group vs. $8.9 \%$ in the placebo group ( $\mathrm{HR}=0.74 ; 95 \% \mathrm{CI}=0.58-0.95 ; \mathrm{P}<0.001$ for noninferiority)

Secondary: Nonfatal MI occurred in $2.9 \%$ of patients receiving semaglutide vs. $3.9 \%$ of those receiving placebo $(\mathrm{HR}=0.74 ; 95 \%$

$\mathrm{CI}=0.51-1.08 ; P=0.12)$; nonfatal stroke occurred in $1.6 \%$ and $2.7 \%$, respectively $(\mathrm{HR}=0.61 ; 95 \% \mathrm{CI}=0.38-0.99 ; P=0.04)$; expanded composite CV outcome: $12.1 \%$ with semaglutide vs. $16.0 \%$ with placebo (HR=0.74; $95 \% \mathrm{CI}=0.62-0.89$ ); the following were not significantly different between groups $(P \geq 0.05)$ : death from any cause, death from CV cause, nonfatal MI, hospitalization for unstable angina, hospitalization for heart failure

\begin{tabular}{l|l}
\hline Conclusions & The rate of MACE was significantly lower in patients receiving semaglutide compared with those receiving placebo and confirmed the
\end{tabular} noninferiority of semaglutide

Alc=glycated hemoglobin; $C I=$ confidence interval; $C V=$ cardiovascular; GLP-1 RA=glucagon-like peptide-1 receptor agonist; HR=hazard ratio; $M A C E=$ major adverse cardiovascular event; $M I=$ myocardial infarction; $O A D=$ oral antidiabetic drug; $Q W=$ once weekly; T2DM = type 2 diabetes mellitus; TBD=to be determined.

the rate of hospitalization for heart failure, or the rate of death among patients with T2DM who had experienced acute coronary syndrome within 180 days before screening. ${ }^{55}$ In addition, recently published large meta-analyses of data from GLP-1 RA studies have identified potential benefits with regard to all-cause mortality, cardiovascular mortality, and MACE risk, although quality and conclusions of meta-analyses are not uniform, and there are likely differences between individual GLP-1 RAs. ${ }^{56-60}$
Once-weekly GLP-1 RAs are increasing in use as they provide an efficacious treatment, with lower rates of hypoglycemia and weight gain similar to the daily options but with the added benefit of a decreased injection burden. For this reason, the purpose of this article is to review published cardiovascular outcome data specific to once-weekly GLP-1 RAs, including exenatide ER, dulaglutide, and semaglutide. 


\section{FIGURE 2 Cardiovascular Outcomes with Once-Weekly Exenatide Versus Placebo, EXSCEL Trialo1}

\section{A. Primary Cardiovascular Outcome}

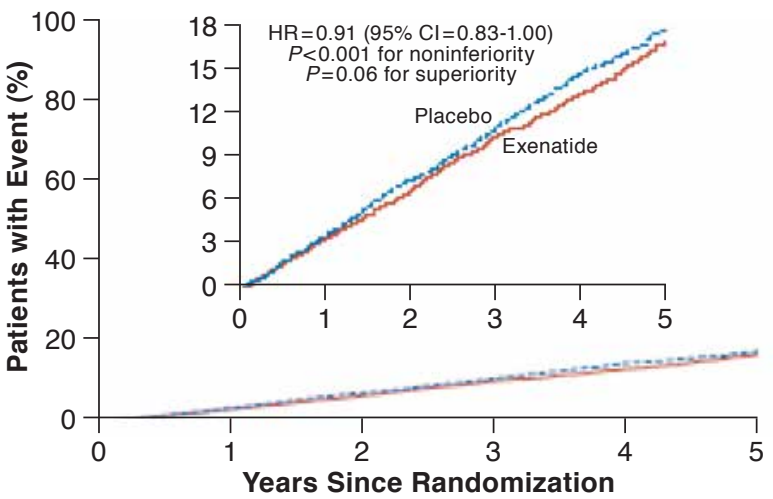

\begin{tabular}{l|l|l|l|l|l|l|l|l|l|l|r|}
\hline At Risk, $\mathbf{n}$ \\
\hline Placebo & 7,396 & 7,120 & 6,897 & 6,565 & 5,908 & 4,468 & 3,565 & 2,961 & 2,209 & 1,366 & 687 \\
\hline Exenatide & 7,356 & 7,101 & 6,893 & 6,580 & 5,912 & 4,475 & 3,595 & 3,053 & 2,281 & 1,417 & 727 \\
\hline
\end{tabular}

\section{Death from Cardiovascular Causes}

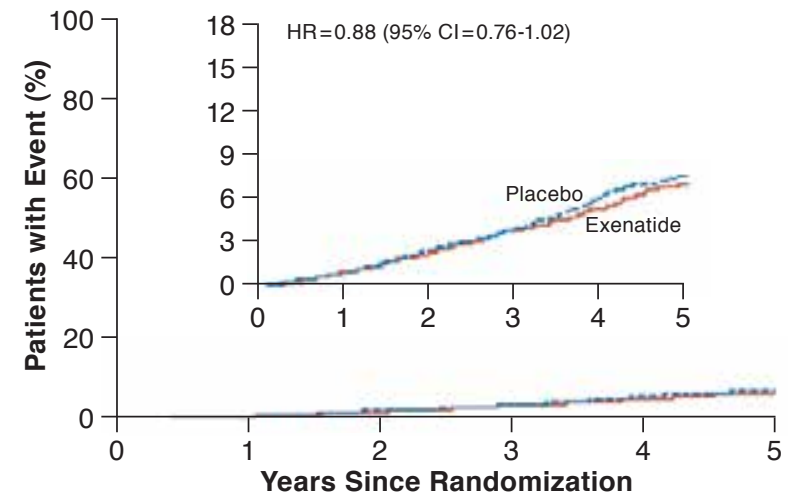

\begin{tabular}{|l|l|l|l|l|l|l|l|l|l|l|r|}
\hline At Risk, $\mathbf{n}$ \\
\hline Placebo & 7,396 & 7,344 & 7,278 & 7,058 & 6,470 & 5,019 & 4,091 & 3,478 & 2,666 & 1,695 & 907 \\
\hline Exenatide & 7,356 & 7,304 & 7,234 & 7,028 & 6,433 & 4,991 & 4,095 & 3,518 & 2,698 & 1,726 & 892 \\
\hline
\end{tabular}

\section{B. Death from Any Cause}

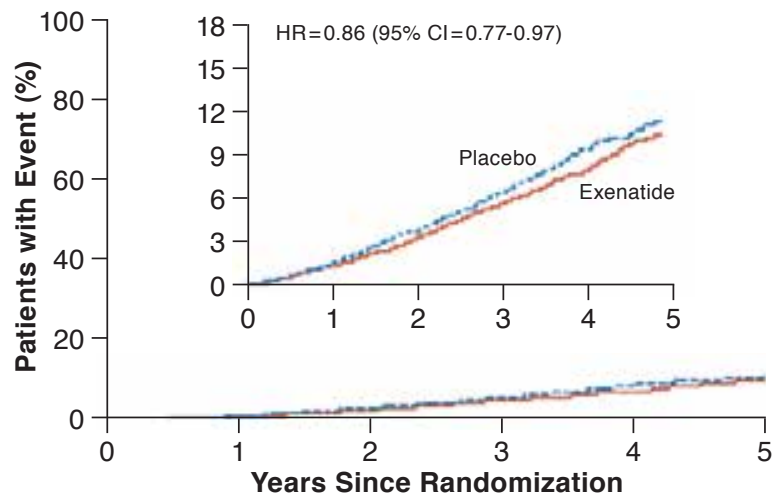

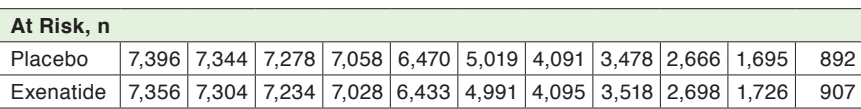

\section{Hospitalization for Heart Failure}

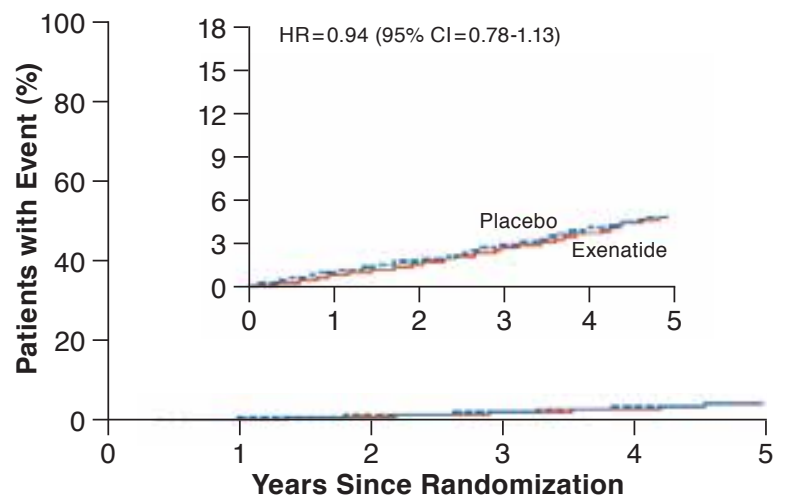

At Risk, $n$

\begin{tabular}{|l|r|r|r|r|r|r|r|r|r|r|r|}
\hline Placebo & 7,396 & 7,183 & 7,019 & 6,743 & 6,112 & 4,678 & 3,756 & 3,156 & 2,375 & 1,464 & 735 \\
\hline
\end{tabular}

\begin{tabular}{|l|l|l|l|l|l|l|l|l|l|l|l|l|} 
Exenatide & 7,356 & 7,174 & 7,023 & 6,756 & 6,108 & 4,669 & 3,790 & 3,234 & 2,430 & 1,517 & 776 \\
\hline
\end{tabular}

From Holman RR, Bethel MA, Mentz RJ, et al. Effects of once-weekly exenatide on cardiovascular outcomes in type 2 diabetes. N Engl J Med. 2017;377(13):1228-39. Copyright (C) 2017 Massachusetts Medical Society. Reprinted with permission from Massachusetts Medical Society.

Note: This figure shows the rates of the primary cardiovascular outcome (a composite of death from cardiovascular causes, nonfatal myocardial infarction, or nonfatal stroke), death from any cause, death from cardiovascular causes, and hospitalization for heart failure in the exenatide and placebo groups. The inset in each panel shows the same data on an enlarged $y$-axis.

$C I=$ confidence interval; $H R=$ hazard ratio.

\section{Prospective Cardiovascular Outcome Trials} with Once-Weekly GLP-1 RAs

To date, there are 2 completed and published cardiovascular outcome trials that were designed to evaluate MACE with once-weekly GLP-1 RAs (EXenatide Study of Cardiovascular Event Lowering [EXSCEL], ${ }^{61}$ SUSTAIN-6, ${ }^{50}$ Table 2). An additional study, Researching cardiovascular Events with a Weekly INcretin in Diabetes (REWIND ${ }^{62}$ ), is ongoing.
EXSCEL was a phase 3, double-blind, randomized, placebocontrolled study that assessed the long-term cardiovascular safety and efficacy of once-weekly exenatide ER $2 \mathrm{mg}$ in patients with T2DM who had a wide range of cardiovascular risk (Table 2). ${ }^{50,61,62}$ Unlike SUSTAIN-6, this study was designed and powered to evaluate both noninferiority and superiority versus placebo, and therefore nonsignificant results cannot be attributed to insufficient power. Overall, 14,752 patients (of whom 10,782 [73.1\%] had previous cardiovascular 


\section{FIGURE 3 Four-Component MACE Endpoints from Meta-Analysis of 9 Randomized Clinical Trials} with Dulaglutide ${ }^{63}$

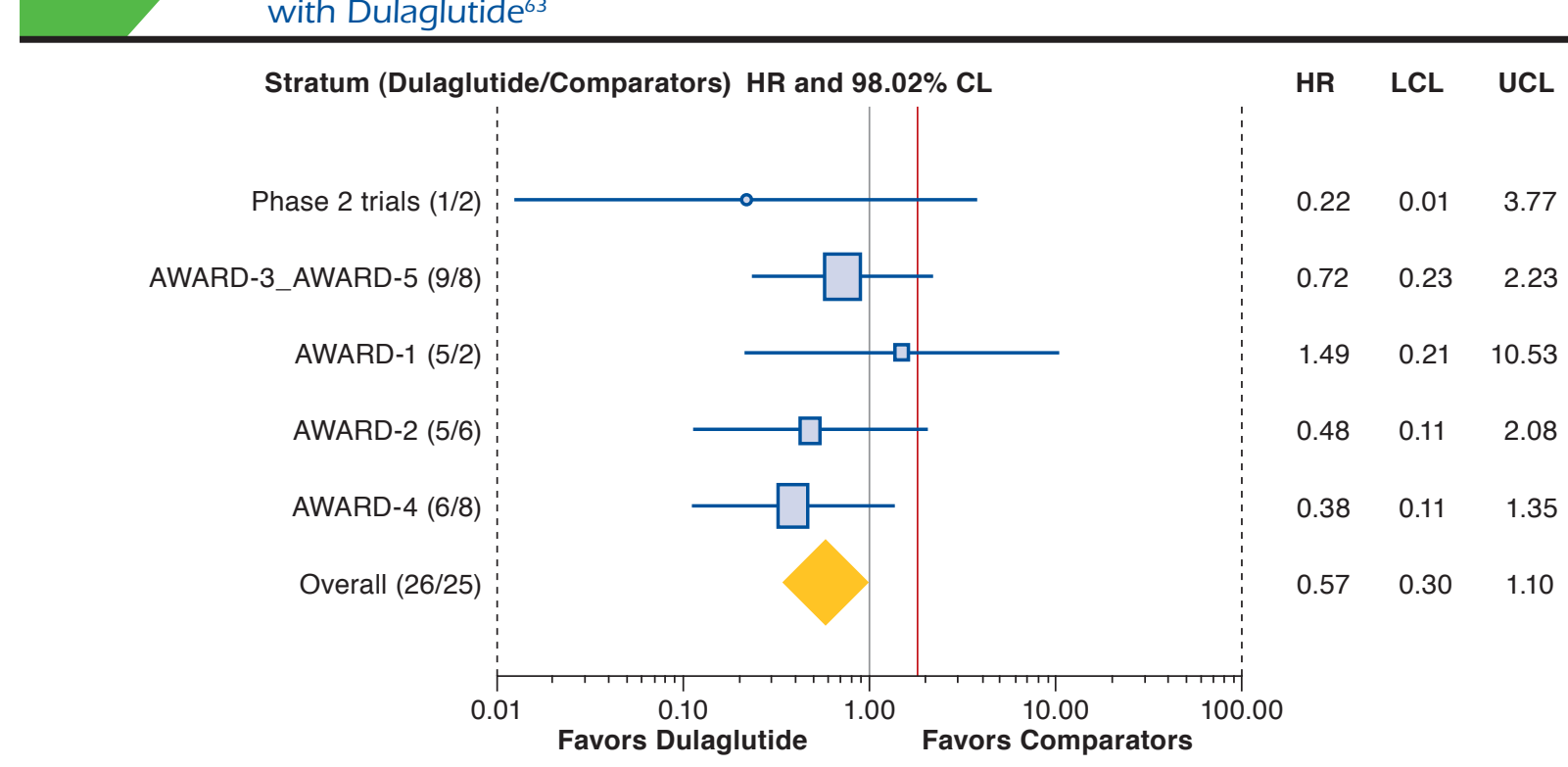

From Ferdinand K, Botras K, Atisso C, Sager PT. Cardiovascular safety for once-weekly dulaglutide in type 2 diabetes: a pre-specified meta analysis of prospectively

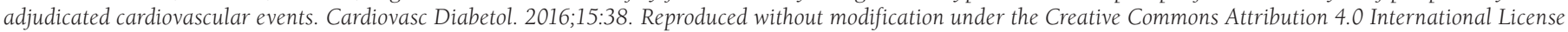
(https://creativecommons.org/publicdomain/zero/1.0/).

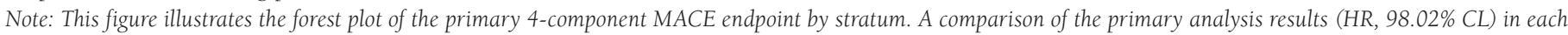

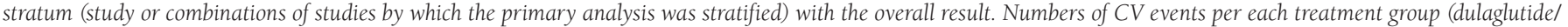
comparators) are indicated by the parentheses in the y-axis under Stratum.

$C L=$ confidence limit; $C V=$ cardiovascular; HR=hazard ratio; $L C L=$ lower confidence limit; MACE= major adverse cardiac event; UCL= upper confidence limit.

disease [CVD]) were followed for a median of 3.2 years. Based on the primary outcome of MACE (death from cardiovascular causes, nonfatal MI, or nonfatal stroke), which occurred in $11.4 \%$ of exenatide-treated patients and $12.2 \%$ of placebotreated patients ( $\mathrm{HR}=0.91 ; 95 \% \mathrm{CI}=0.83-1.00)$, exenatide was noninferior to placebo with respect to safety $(P<0.001)$ but was not superior to placebo with respect to efficacy despite trending lower $(P=0.06$; Figure 2$)$. There were also no significant differences between exenatide ER and placebo with regard to risk of death from any cause $(\mathrm{HR}=0.86$; 95\% CI=0.77-0.97; nonsignificant on basis of the hierarchical testing plan), fatal or nonfatal MI ( $\mathrm{HR}=0.97 ; 95 \% \mathrm{CI}=0.85-1.10)$, fatal or nonfatal stroke ( $\mathrm{HR}=0.85 ; 95 \% \mathrm{CI}=0.70-1.03)$, hospitalization for heart failure $(H R=0.94 ; 95 \% C I=0.78-1.13)$, and hospitalization for acute coronary syndrome $(\mathrm{HR}=1.05 ; 95 \% \mathrm{CI}=0.94-1.18)$.

The REWIND trial is an ongoing (at the time of manuscript development) phase 3, double-blind, randomized, placebocontrolled study designed to assess the effects of once-weekly dulaglutide $1.5 \mathrm{mg}$ on the incidence of cardiovascular outcomes. ${ }^{62}$ The patient population consists of patients aged $\geq 50$ years with $\mathrm{T} 2 \mathrm{DM}, \mathrm{Alc} \leq 9.5 \%$, and, depending on age category ( $\geq 50$ years, $\geq 55$ years, or $\geq 60$ years), either a prior cardiovascular event, evidence of cardiovascular disorder, or at least 2 other cardiovascular risk factors, such as tobacco use, dyslipidemia, or hypertension. The primary cardiovascular outcome is the first occurrence of MACE. Secondary outcomes include cardiovascular death, nonfatal MI, nonfatal stroke, a composite outcome of retinal or renal disease, hospitalization for unstable angina, heart failure requiring hospitalization or an urgent heart failure visit, and all-cause mortality. Based on the ClinicalTrial.gov listing as of January 18, 2018, the study has enrolled an estimated 9,622 patients, with an average duration of follow-up of 6.5 years. The anticipated completion data for REWIND is July 2018.

Because the REWIND trial results are not yet available, the results of a meta-analysis of cardiovascular outcomes from safety and efficacy trials for dulaglutide are included in this review. The large $(\mathrm{N}=6,010)$ meta-analysis examined the cardiovascular risk of dulaglutide from 9 randomized phase 2 and 3 clinical efficacy and safety trials ranging from 12 to 104 weeks in duration (mean treatment duration, 333 days). ${ }^{63}$ Baseline cardiovascular risks were similar between dulaglutide and comparator groups. A composite MACE endpoint of death due to cardiovascular causes, nonfatal MI, nonfatal stroke, or hospitalization for unstable angina occurred in $0.67 \%$ of patients in the dulaglutide group compared with $1.18 \%$ in the comparator 


\section{FIGURE 4 Cardiovascular Outcomes with Once-Weekly Semaglutide Versus Placebo, SUSTAIN-6 Trial50}

\section{A. Primary Outcome}

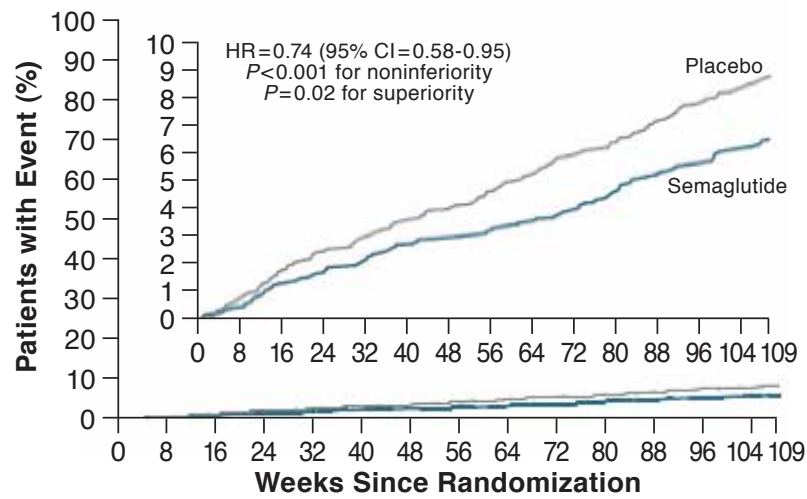

\begin{tabular}{|l|c|c|c|c|c|c|c|}
\hline \multicolumn{1}{|l|}{ At Risk, $\mathbf{n}$} \\
\hline Placebo & 1,649 & 1,616 & 1,586 & 1,567 & 1,534 & 1,508 & 1,479 \\
\hline Semaglutide & 1,648 & 1,619 & 1,601 & 1,584 & 1,568 & 1,543 & 1,524 \\
\hline
\end{tabular}

\section{Nonfatal Stroke}

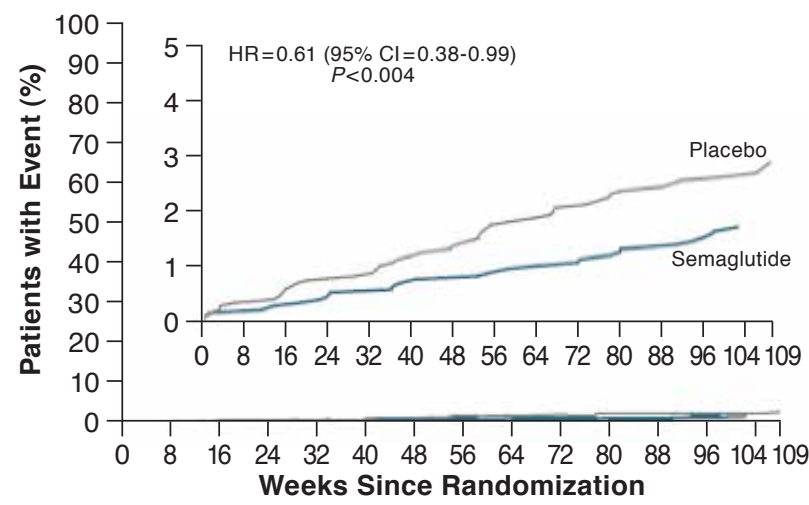

\begin{tabular}{l|l|l|l|l|l|l|l|}
\hline At Risk, $\mathbf{n}$ \\
\hline Placebo & 1,649 & 1,629 & 1,611 & 1,597 & 1,571 & 1,548 & 1,528 \\
\hline Semaglutide & 1,648 & 1,630 & 1,619 & 1,606 & 1,593 & 1,572 & 1,558 \\
\hline
\end{tabular}

\section{B. Nonfatal Myocardial Infarction}

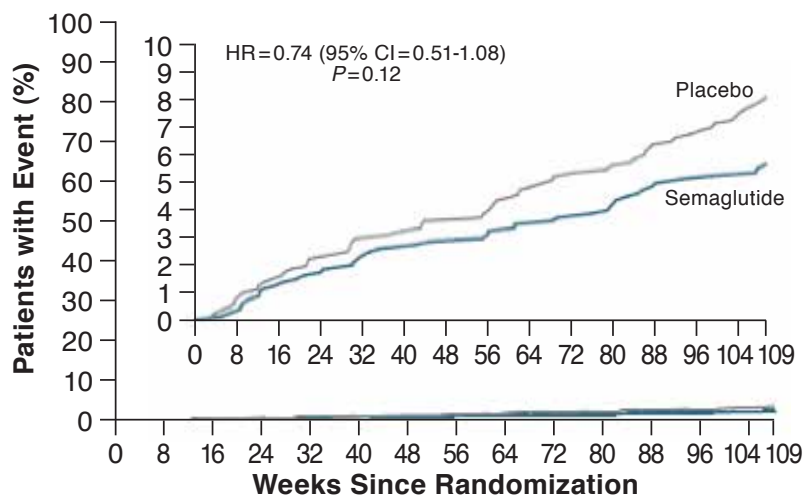

\begin{tabular}{|l|l|l|l|l|l|l|l|}
\hline \multicolumn{1}{|l|}{ At Risk, $\mathbf{n}$} \\
\hline Placebo & 1,649 & 1,624 & 1,598 & 1,587 & 1,562 & 1,542 & 1,516 \\
\hline Semaglutide & 1,648 & 1,623 & 1,609 & 1,595 & 1,582 & 1,560 & 1,543 \\
\hline
\end{tabular}

\section{Death from Cardiovascular Causes}

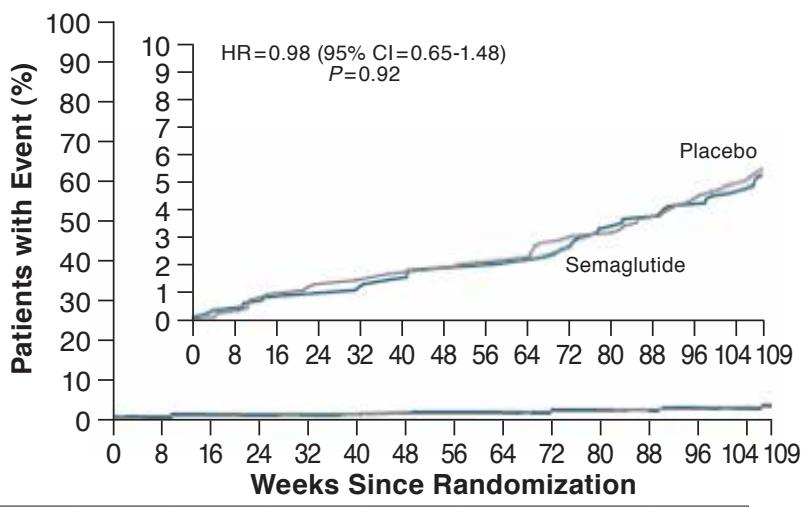

\begin{tabular}{|l|c|c|c|c|c|c|c|}
\hline \multicolumn{1}{|l|}{ At Risk, $\mathbf{n}$} \\
\hline Placebo & 1,649 & 1,637 & 1,623 & 1,617 & 1,600 & 1,584 & 1,566 \\
\hline Semaglutide & 1,648 & 1,634 & 1,627 & 1,617 & 1,607 & 1,589 & 1,579 \\
\hline
\end{tabular}

From Marso S, Bain S, Consoli A, et al. Semaglutide and cardiovascular outcomes in patients with type 2 diabetes. N Engl J Med. 2016;375(19):1834-44.

Copyright () 2016 Massachusetts Medical Society. Reprinted with permission from Massachusetts Medical Society.

Note: This figure shows the Kaplan-Meier plots of the primary outcome (a composite of cardiovascular death, nonfatal myocardial infarction, or nonfatal stroke; Panel A), nonfatal myocardial infarction (Panel B), nonfatal stroke (Panel C), and death from cardiovascular causes (Panel D). The trial included a planned observation period of 109 weeks for all patients (a 104-week treatment period with a 5-week follow-up period). In Panel C, there were no events in the semaglutide group after week 104. Insets show the same data on an expanded $y$-axis.

group (active treatment or placebo). The estimated HR for dulaglutide versus comparators was 0.57 , with an adjusted $98.02 \%$ confidence limit $(\mathrm{CL})$ of $0.30-1.10(P=0.046)$, indicating no significant difference between treatment groups (Figure 3). The relative risk of experiencing a nonfatal MI was significantly lower in the dulaglutide group versus the comparator group (estimated $\mathrm{HR}=0.35$; adjusted $98.02 \% \mathrm{CL}=0.13-0.95$; $P=0.014)$. There were no significant differences between dula- glutide and comparator groups with regard to the risk of death due to cardiovascular causes, nonfatal stroke, or hospitalization for unstable angina. Thus, the authors concluded that dulaglutide does not increase the risk of MACE in patients with T2DM based on the meta-analysis findings.

SUSTAIN-6 was a 2-year, phase 3, premarketing, double-blind, randomized, placebo-controlled trial specifically designed to evaluate the cardiovascular safety of semaglutide 
(0.5 $\mathrm{mg}$ and $1.0 \mathrm{mg}$ ) compared with placebo in accordance with regulatory guidelines (Table 2)..$^{50}$ The study enrolled 3,297 patients aged 50 years and older with T2DM and established ASCVD, with an ASCVD risk equivalent ( $\geq$ stage 3 chronic kidney disease [CKD]) or at high risk of ASCVD (defined as having at least 1 of the following: persistent microalbuminuria or proteinuria; hypertension and left ventricular hypertrophy by electrocardiogram or imaging; left ventricular systolic or diastolic dysfunction by imaging; or ankle/brachial index less than 0.9$) ; 83 \%$ of patients had established CVD, $\geq$ stage 3 CKD, or both. The composite primary outcome of MACE (death from cardiovascular causes, nonfatal MI, or nonfatal stroke) occurred in $6.6 \%$ of semaglutide-treatment patients versus $8.9 \%$ of those in the placebo group ( $\mathrm{HR}=0.74 ; 95 \% \mathrm{CI}=0.58$ 0.95 ; noninferiority achieved, $P<0.001$; superiority achieved, $P=0.02$; Figure 4). Compared with placebo, semaglutide was associated with a significant reduction in nonfatal stroke $(1.6 \%$ vs. $2.7 \%, \mathrm{HR}=0.61 ; 95 \% \mathrm{CI}=0.38-0.99 ; P=0.04)$ and no difference in nonfatal MI $(2.9 \%$ vs. $3.9 \%$, HR $=0.74 ; 95 \% \mathrm{CI}=0.51$ 1.08; $P=0.12$ ). There were no significant differences between semaglutide and placebo for individual outcomes, including hospitalization for heart failure or death from any cause or cardiovascular causes. However, because this was a safety trial and the primary hypothesis was for noninferiority relative to placebo, the study was not statistically powered to demonstrate superiority.

\section{Health Economic Implications}

Cardiovascular-related disease and associated conditions and risk factors are the primary drivers of direct and indirect costs of diabetes. ${ }^{2}$ While there are no data specifically evaluating cost implications relating to $\mathrm{CV}$ risks and events in patients treated with once-weekly GLP-1 RAs, several studies have quantified the impact of MACE on costs in patients with T2DM. ${ }^{64-68} \mathrm{~A}$ retrospective claims database analysis using data representative of a large U.S. managed care population estimated that the mean cost of initial hospitalization (adjusted to 2018 dollars) for patients with T2DM was $\$ 28,689$ for MI and $\$ 13,005$ for stroke. ${ }^{69}$ These costs were only slightly higher than corresponding costs estimated for patients without T2DM who had these events. However, patients with T2DM had significantly higher mean cumulative total direct costs per patient for cardiovascular events over 3 years of follow-up. Ward et al. (2014) used multiple sources (inpatient and emergency databases, national physician and laboratory fee schedules, published literature, and government reports) to estimate direct medical costs of diabetes complications, including acute event and follow-up care costs over 1 year, for U.S. health care payers. ${ }^{64}$ Estimated acute event and follow-up care costs over 1 year were $\$ 60,937$ per patient for MI and $\$ 45,471$ per patient for ischemic stroke (adjusted to 2018 dollars).
Li et al. (2013) analyzed data from patient surveys, chart reviews, and health insurance claims from 7,109 patients with T2DM participating in the Translating Research Into Action for Diabetes (TRIAD) study between 1999 and 2002 to evaluate medical costs associated with T2DM complications and comorbidities. ${ }^{65}$ Among patients with T2DM, estimated annualized medical costs were about $80 \%$ higher in the presence of coronary heart disease and about 35\% higher in the presence of a cerebrovascular accident. These findings highlight the increased medical costs in patients with T2DM that are related to cardiovascular and cerebrovascular complications.

Cardiovascular events occurring in patients with T2DM contribute substantially to per-member health care costs in Medicare members with T2DM and in the overall Medicare population. A claims-based actuarial analysis of a randomly generated subset of 2013 and 2014 Medicare data found that annual rates for MI, stroke, unstable angina admission, and coronary revascularization in the T2DM population were 3.3 , $2.4,3.2$, and 2.8 times higher, respectively, than in the nonT2DM population. ${ }^{66}$ Although patients with T2DM accounted for only $22 \%$ of the Medicare sample, half of the cardiovascular event costs for the entire Medicare sample (i.e., patients with or without T2DM) were due to patients with T2DM. Additionally, $3.6 \%$ of the total per-member per-month (PMPM) cost for the overall Medicare sample was attributable to cardiovascular events occurring in patients with T2DM. After adjustments to account for underlying differences in demographics and morbidity between patients with and without cardiovascular events, costs associated with cardiovascular events occurring in the T2DM population were found to represent $18.1 \%$ of the total PMPM cost ( $\$ 353$ of $\$ 1,951$, adjusted to 2018 dollars) for patients with T2DM and $6.9 \%$ of the PMPM cost (\$78 of $\$ 1,132)$ in the overall Medicare sample.

Consideration of cardiovascular risk factors and the potential modifying effects of medications is important when evaluating the economic impact of MACE in patients with T2DM A U.S. administrative claims data study evaluated cardiovascular event costs in commercially, Medicare Supplemental-, and Medicaid-insured patients with T2DM. ${ }^{67}$ The sample included 1,415,598 patients with T2DM who were classified into 3 cardiovascular risk groups (highest, medium, and lowest) based on age and comorbidities. Patients were followed over a 1-year period (July 2010-June 2011), and those with MACE (death or nonfatal MI or nonfatal stroke) were additionally followed for up to 1 year after the initial event to determine longitudinal costs. Multivariable regression was used to compare costs between patients with and without MACE. Overall, 10,399 patients experienced MACE. The expected mean PMPM costs of MACE per 100 covered patients generally increased with CVD risk and varied by payer; costs (adjusted to 2018 dollars) ranged from $\$ 1,714$ in the lowest-risk commercially insured patients to $\$ 20,636$ in the highest-risk 
Medicaid-insured patients. The mean longitudinal costs among patients with T2DM experiencing MACE ranged from \$24,966 for the initial event and $\$ 2,742$ PMPM for up to 1 year of followup care in Medicare Supplemental-insured patients with stroke to $\$ 45,736$ for the initial event and $\$ 5,288$ PMPM for up to 1 year of follow-up care among Medicaid-insured patients with MI.

\section{Summary and Conclusions}

The benefits of GLP-1 RAs appear to extend beyond reducing blood glucose and weight to positively affecting a range of atherosclerotic risk factors. Placebo-controlled studies to date that have looked specifically at the risk of MACE with onceweekly GLP-1 RAs found no increased risk with exenatide ER and a significant reduction of MACE events with semaglutide. Findings from the ongoing REWIND trial with dulaglutide are anticipated and may shed further light on the impact of once-weekly GLP-1 RAs on cardiovascular outcomes. When interpreting data from these clinical trials, it is important to consider differences in study populations and statistical design limitations. Choices of diabetes treatments have evolved to the point where broader health impacts beyond simple blood glucose regulation are relevant considerations and could reduce substantial costs of MACE in patients with T2DM. The potential for reducing cardiovascular events in patients with T2DM has obvious health benefits but, beyond that, may potentially translate into cost savings. Future studies are needed to assess the impact of long-term treatment with once-weekly GLP-1 RAs on CVD-related costs among patients with T2DM in the managed care setting.

\section{Authors}

MICHAEL HEILE, MD, FAAFP, TriHealth, Cincinnati, Ohio; KATHLEEN WYNE, MD, PhD, FACE, FNLA, The Ohio State University Wexner Medical Center, Columbus; LIANA K. BILLINGS, MD, MMSc, NorthShore University Health System, Skokie, Illinois; ANTHONY CANNON, MD, FACE, Private Practice, Hamilton, New Jersey; YEHUDA HANDELSMAN, MD, FACP, FNLA, FASPC, MACE, Metabolic Institute of America, Tarzana, California; and MICHAEL SHANNON, MD, PMG Olympia Endocrinology, Lacey, Washington.

\section{DISCLOSURES}

This supplement was funded by Novo Nordisk. Heile reports speaker fees from and has served as advisor to Novo Nordisk. Billings reports personal fees from Dexcom, Novo Nordisk, and Sanofi. Cannon reports speaker fees and owns stock in Novo Nordisk. Handelsman reports research grants from Amgen, AstraZeneca, Bristol-Myers Squibb, Boehringer Ingelheim, Grifols, Janssen, Lexicon, Merck, Novo Nordisk, Regeneron, and Sanofi; speaker fees from Amarin, Amgen, AstraZeneca, Boehringer Ingelheim-Lilly, Janssen, Merck, Novo Nordisk, Regeneron, and Sanofi; and has served in advisory capacity to Amarin, Amgen, AstraZeneca, Boehringer Ingelheim, Eisai, Intarcia, Janssen, Lilly, Merck, Merck-Pfizer, Novo Nordisk, Regeneron, and Sanofi. Shannon reports consultant and speaker fees from Novo Nordisk and Boehringer Ingelheim-Lilly Alliance. Wyne has nothing to disclose.

\section{ACKNOWLEDGMENTS}

Writing assistance was provided by Adrienne Drinkwater, $\mathrm{PhD}$, and Sandra Westra, PharmD, through Churchill Communications (Maplewood, NJ) and funded by Novo Nordisk

\section{REFERENCES}

1. Dalsgaard N, Bronden A, Vilsboll T, Knop FK. Cardiovascular safety and benefits of GLP-1 receptor agonists. Expert Opin Drug Saf. 2017;16(3):351-63.

2. American Diabetes Association. Cardiovascular disease and risk management. Sec. 9. In: Standards of Medical Care in Diabetes 2018. Diabetes Care. 2018;41(Suppl 1):S86-104. Available at: http://care.diabetesjournals.org/content/41/Supplement_1/S86. Accessed July 10, 2018.

3. UK Prospective Diabetes Study (UKPDS) Group. Intensive blood glucose control with sulphonylureas or insulin compared with conventional treatment and risk of complications in patients with type 2 diabetes (UKPDS 33). Lancet. 1998;352(9131):837-53

4. Holman RR, Paul SK, Bethel MA, Matthews DR, Neil HA. 10-year follow-up of intensive glucose control in type 2 diabetes. N Engl J Med. 2008;359(15):1577-89.

5. ADVANCE Collaborative Group, Patel A, MacMahon S, et al. Intensive blood glucose control and vascular outcomes in patients with type 2 diabetes. N Engl J Med. 2008;358(24):2560-72.

6. Action to Control Cardiovascular Risk in Diabetes Study Group, Gerstein HC, Miller ME, et al. Effects of intensive glucose lowering in type 2 diabetes. N Engl J Med. 2008;358(24):2545-59.

7. Duckworth W, Abraira C, Moritz T, et al. Glucose control and vascular complications in veterans with type 2 diabetes. N Engl J Med. 2009;360(2): 129-39.

8. Gaede P, Vedel P, Larsen N, Jensen GV, Parving HH, Pedersen O. Multifactorial intervention and cardiovascular disease in patients with type 2 diabetes. N Engl J Med. 2003;348(5):383-93.

9. Nissen SE, Wolski K. Effect of rosiglitazone on the risk of myocardial infarction and death from cardiovascular causes. N Engl J Med. 2007;356(24):2457-71.

10. Home PD, Pocock SJ, Beck-Nielsen H, et al. Rosiglitazone evaluated for cardiovascular outcomes in oral agent combination therapy for type 2 diabetes (RECORD): a multicentre, randomised, open-label trial. Lancet. 2009;373(9681):2125-35

11. U.S. Department of Health and Human Services, Food and Drug Administration, Center for Drug Evaluation and Research (CDER). Guidance for industry: diabetes mellitus-evaluating cardiovascular risk in new antidiabetic therapies to treat type 2 diabetes. December 2008. Available at: https://www.fda.gov/downloads/Drugs/Guidances/ucm071627.pdf. Accessed July 10, 2018.

12. Lee S, Lee DY. Glucagon-like peptide-1 and glucagon-like peptide-1 receptor agonists in the treatment of type 2 diabetes. Ann Pediatr Endocrinol Metab. 2017;22(1):15-26.

13. Levin PA, Nguyen H, Wittbrodt ET, Kim SC. Glucagon-like peptide-1 receptor agonists: a systematic review of comparative effectiveness research. Diabetes Metab Syndr Obes. 2017;10:123-39.

14. Doyle ME, Egan JM. Mechanisms of action of glucagon-like peptide 1 in the pancreas. Pharmacol Ther. 2007;113(3):546-93.

15. Byetta (exenatide extended-release for injectable suspension). AstraZeneca Pharmaceuticals. Revised February 2015. Available at: https://www.fda.gov/ downloads/drugs/drugsafety/ucm191084.pdf. Accessed July 10, 2018.

16. Victoza (liraglutide [rDNA origin] injection), solution for subcutaneous use. Novo Nordisk. Revised August 2017. Available at: https://www.accessdata.fda.gov/drugsatfda_docs/label/2017/022341s027lbl.pdf. Accessed July 23, 2018. 
17. Adlyxin (lixisenatide) injection, for subcutaneous use. Sanofi-Aventis U.S. Revised July 2016. Available at: https://www.accessdata.fda.gov/drugsatfda_docs/nda/2016/208471Origls000lbl.pdf. Accessed July 10, 2018.

18. Bydureon (exenatide extended-release) injectable. AstraZeneca Pharmaceuticals. Revised September 2015. Available at: https://www.accessdata.fda.gov/drugsatfda_docs/label/2015/022200s020lbl.pdf. Accessed July 23, 2018

19. Trulicity (dulaglutide) injection, for subcutaneous use. Eli Lilly and Company. Revised January 2017. Available at: https://www.accessdata.fda.gov/ drugsatfda_docs/label/2017/125469s011s013lbl.pdf. Accessed July 10, 2018.

20. Ozempic (semaglutide) injection, for subcutaneous use. Novo Nordisk. Revised December 2017. Available at: https://www.accessdata.fda.gov/drugsatfda_docs/label/2017/209637lbl.pdf. Accessed July 10, 2018.

21. Garber AJ, Abrahamson MJ, Barzilay JI, et al. Consensus statement by the American Association of Clinical Endocrinologists and American College of Endocrinology on the comprehensive type 2 diabetes management algorithm-2018 executive summary. Endocr Pract. 2018;24(1):91-120.

22. Drucker DJ, Buse JB, Taylor K, et al. Exenatide once weekly versus twice daily for the treatment of type 2 diabetes: a randomised, open-label, noninferiority study. Lancet. 2008;372(9645):1240-50

23. Bergenstal RM, Wysham C, Macconell L, et al. Efficacy and safety of exenatide once weekly versus sitagliptin or pioglitazone as an adjunct to metformin for treatment of type 2 diabetes (DURATION-2): a randomised trial. Lancet. 2010;376(9739):431-39.

24. Diamant M, Van Gaal L, Stranks S, et al. Once weekly exenatide compared with insulin glargine titrated to target in patients with type 2 diabetes (DURATION-3): an open-label randomised trial. Lancet. 2010;375(9733):2234-43.

25. Russell-Jones D, Cuddihy RM, Hanefeld M, et al. Efficacy and safety of exenatide once weekly versus metformin, pioglitazone, and sitagliptin used as monotherapy in drug-naive patients with type 2 diabetes (DURATION-4): a 26-week double-blind study. Diabetes Care. 2012;35(2):252-58.

26. Blevins T, Pullman J, Malloy J, et al. DURATION-5: exenatide once weekly resulted in greater improvements in glycemic control compared with exenatide twice daily in patients with type 2 diabetes. J Clin Endocrinol Metab. 2011;96(5):1301-10.

27. Buse JB, Nauck M, Forst T, et al. Exenatide once weekly versus liraglutide once daily in patients with type 2 diabetes (DURATION-6): a randomised, open-label study. Lancet. 2013;381:117-24.

28. Guja C, Frias JP, Somogyi A, et al. Effect of exenatide QW or placebo, both added to titrated insulin glargine, in uncontrolled type 2 diabetes: the DURATION-7 randomized study. Diabetes Obes Metab. 2018;20(7):1602-14.

29. Frías JP, Guja C, Hardy E, et al. Exenatide once weekly plus dapagliflozin once daily versus exenatide or dapagliflozin alone in patients with type 2 diabetes inadequately controlled with metformin monotherapy (DURATION-8): a 28 week, multicentre, double-blind, phase 3, randomised controlled trial. Lancet Diabetes Endocrinol. 2016;4(12):1004-16.

30. Wysham C, Blevins T, Arakaki R, et al. Efficacy and safety of dulaglutide added onto pioglitazone and metformin versus exenatide in type 2 diabetes in a randomized controlled trial (AWARD-1). Diabetes Care. 2014;37:2159-67.

31. Umpierrez G, Tofé Povedano S, Pérez Manghi F, Shurzinske L, Pechtner $\mathrm{V}$. Efficacy and safety of dulaglutide monotherapy versus metformin in type 2 diabetes in a randomized controlled trial (AWARD-3). Diabetes Care. 2014;37(8):2168-76.

32. Blonde L, Jendle J, Gross J, et al. Once-weekly dulaglutide versus bedtime insulin glargine, both in combination with prandial insulin lispro, in patients with type 2 diabetes (AWARD-4): a randomised, open-label, phase 3, non-inferiority study. Lancet. 2015;385(9982):2057-66.

33. Nauck M, Weinstock RS, Umpierrez GE, et al. Efficacy and safety of dulaglutide versus sitagliptin after 52 weeks in type 2 diabetes in a randomized controlled trial (AWARD-5). Diabetes Care. 2014;37(8):2149-58.
34. Dungan KM, Povedano ST, Forst T, et al. Once-weekly dulaglutide versus once-daily liraglutide in metformin-treated patients with type 2 diabetes (AWARD-6): a randomised, open-label, phase 3, non-inferiority trial. Lancet. 2014;384(9951):1349-57.

35. Dungan KM, Weitgasser R, Perez Manghi F, et al. A 24-week study to evaluate the efficacy and safety of once-weekly dulaglutide added on to glimepiride in type 2 diabetes (AWARD-8). Diabetes Obes Metab. 2016;18(5):475-82.

36. Pozzilli P, Norwood P, Jódar E, et al. Placebo-controlled, randomized trial of the addition of once-weekly glucagon-like peptide-1 receptor agonist dulaglutide to titrated daily insulin glargine in patients with type 2 diabetes (AWARD-9). Diabetes Obes Metab. 2017;19(7):1024-31.

37. Sorli C, Harashima SI, Tsoukas GM, et al. Efficacy and safety of onceweekly semaglutide monotherapy versus placebo in patients with type 2 diabetes (SUSTAIN 1): a double-blind, randomised, placebo-controlled, parallel-group, multinational, multicentre phase 3 a trial. Lancet Diabetes Endocrinol. 2017;5(4):251-60.

38. Aroda VR, Bain SC, Cariou B, et al. Efficacy and safety of once-weekly semaglutide versus once-daily insulin glargine as add-on to metformin (with or without sulfonylureas) in insulin-naive patients with type 2 diabetes (SUSTAIN 4): a randomised, open-label, parallel-group, multicentre, multinational, phase 3a trial. Lancet Diabetes Endocrinol. 2017;5(5):355-66.

39. Rodbard H, Lingvay I, Reed J, et al. Efficacy and safety of semaglutide once-weekly vs placebo as add-on to basal insulin alone or in combination with metformin in subjects with type 2 diabetes (SUSTAIN 5). European Foundation for the Study of Diabetes (EASD), Berlin, Germany; September 12-16, 2016 [Abstract 766].

40. Buse JB, Drucker DJ, Taylor KL, et al. DURATION-1: Exenatide once weekly produces sustained glycemic control and weight loss over 52 weeks. Diabetes Care. 2010;33:1255-61.

41. MacConell L, Pencek R, Li Y, Maggs D, Porter L. Exenatide once weekly: sustained improvement in glycemic control and cardiometabolic measures through 3 years. Diabetes Metab Syndr Obes. 2013;6:31-41.

42. Wysham CH, MacConell LA, Maggs DG, Zhou M, Griffin PS, Trautmann ME. Five-year efficacy and safety data of exenatide once weekly: long-term results from the DURATION-1 randomized clinical trial. Mayo Clin Proc. 2015;90(3):356-65.

43. Henry RR, Klein EJ, Han J, Iqbal N. Efficacy and tolerability of exenatide once weekly over 6 years in patients with type 2 diabetes: an uncontrolled open-label extension of the DURATION-1 Study. Diabetes Technol Ther. 2016;18(11):677-86

44. Diamant M, Van Gaal L, Guerci B, et al. Exenatide once weekly versus insulin glargine for type 2 diabetes (DURATION-3): 3 -year results of an open-label randomised trial. Lancet Diabetes Endocrinol. 2014;2(6):464-73.

45. Guja C, Frías J, Ahmed A, et al. DURATION-8 randomized controlled trial 1-year results: efficacy and safety of once-weekly exenatide (ExQW) plus once-daily dapagliflozin (DAPA) vs. ExQW or DAPA alone. Presented at: American Diabetes Association 77th Scientific Session; San Diego, CA; June 9-13, 2017 [Abstract 141-LB].

46. Giorgino F, Benroubi M, Sun JH, Zimmermann AG, Pechtner V. Efficacy and safety of once-weekly dulaglutide versus insulin glargine in patients with type 2 diabetes on metformin and glimepiride (AWARD-2). Diabetes Care. 2015;38(12):2241-49.

47. Weinstock RS, Guerci B, Umpierrez G, Nauck MA, Skrivanek Z, Milicevic Z. Safety and efficacy of once-weekly dulaglutide versus sitagliptin after 2 years in metformin-treated patients with type 2 diabetes (AWARD-5): a randomized, phase III study. Diabetes Obes Metab. 2015;17(9):849-58.

48. Ahrén B, Masmiquel L, Kumar H, et al. Efficacy and safety of onceweekly semaglutide versus once-daily sitagliptin as an add-on to metformin, thiazolidinediones, or both, in patients with type 2 diabetes (SUSTAIN 2): a 56-week, double-blind, phase 3a, randomised trial. Lancet Diabetes Endocrinol. 2017;5(5):341-54. 
49. Ahmann A, Capehorn M, Charpentier G, et al. Efficacy and safety of once-weekly semaglutide vs exenatide ER after 56 weeks in subjects with type 2 diabetes (SUSTAIN 3). Presented at: American Diabetes Association 76th Scientific Session, New Orleans, LA; June 10-14, 2016 [Abstract 187-OR].

50. Marso S, Bain S, Consoli A, et al. Semaglutide and cardiovascular outcomes in patients with type 2 diabetes. N Engl J Med. 2016;375:1834-44.

51. Ussher JR, Drucker DJ. Cardiovascular actions of incretin-based therapies. Circ Res. 2014;114:1788-803.

52. Marso SP, Daniels GH, Brown-Frandsen K, et al. Liraglutide and cardiovascular outcomes in type 2 diabetes. N Engl J Med. 2016;375(4):311-22.

53. Li J, Zheng J, Wang S, Lau HK, Fathi A, Wang Q. Cardiovascular benefits of native GLP-1 and its metabolites: an indicator for GLP-1-therapy strategies. Front Physiol. 2017;8:15.

54. Holman R, Bethel M, George J, et al. Rationale and design of the exenatide study of cardiovascular event lowering trial (EXSCEL). Am Heart J. 2016;174:103-10.

55. Pfeffer MA, Claggett B, Diaz R, et al. Lixisenatide in patients with type 2 diabetes and acute coronary syndrome. N Engl J Med. 2015;373(23):2247-57.

56. Bethel MA, Patel RA, Merrill P, et al. Cardiovascular outcomes with glucagon-like peptide-1 receptor agonists in patients with type 2 diabetes: a meta-analysis. Lancet Diabetes Endocrinol. 2018;6(2):105-13.

57. Fei Y, Tsoi MF, Kumana CR, Cheung TT, Cheung BMY. Network metaanalysis of cardiovascular outcomes in randomized controlled trials of new antidiabetic drugs. Int J Cardiol. 2018;254:291-96.

58. Gargiulo P, Savarese G, D'Amore C, et al. Efficacy and safety of glucagon-like peptide-1 agonists on macrovascular and microvascular events in type 2 diabetes mellitus: a meta-analysis. Nutr Metab Cardiovasc Dis. 2017;27(12):1081-88

59. Monami M, Zannoni S, Pala L, et al. Effects of glucagon-like peptide-1 receptor agonists on mortality and cardiovascular events: a comprehensive meta-analysis of randomized controlled trials. Int J Cardiol. 2017;240:414-21.
60. Peterson S, Barry A. Effect of glucagon-like peptide-1 receptor agonists on all cause mortality and cardiovascular outcomes: a meta-analysis. Curr Diabetes Rev. 2018;14(3):273-79.

61. Holman RR, Bethel MA, Mentz RJ, et al. Effects of once-weekly exenatide on cardiovascular outcomes in type 2 diabetes. N Engl J Med. 2017;377:1228-39.

62. Gerstein HC, Colhoun HM, Dagenais GR, et al. Design and baseline characteristics of participants in the Researching cardiovascular Events with a Weekly INcretin in Diabetes (REWIND) trial on the cardiovascular effects of dulaglutide. Diabetes Obes Metab. 2018;20(1):42-49.

63. Ferdinand K, Botras K, Atisso C, Sager PT. Cardiovascular safety for once-weekly dulaglutide in type 2 diabetes: a pre-specified meta analysis of prospectively adjudicated cardiovascular events. Cardiovasc Diabetol. 2016;15:38.

64. Ward A, Alvarez P, Vo L, Martin S. Direct medical costs of complications of diabetes in the United States: estimates for event-year and annual state costs (USD 2012). J Med Econ. 2014;17(3):176-83.

65. Li R, Bilik D, Brown MB, et al. Medical costs associated with type 2 diabetes complications and comorbidities. Am J Manag Care. 2013;19(5):421-30.

66. Fitch K, Engel T, Sander S, Kuti E, Blumen H. Cardiovascular event incidence and cost in type 2 diabetes mellitus: a Medicare claims-based actuarial analysis. Curr Med Res Opin. 2017;33(10):1795-801.

67. Johnston SS, Sheehan JJ, Shah M, et al. Cardiovascular event costs in patients with type 2 diabetes mellitus. J Med Econ. 2015;18(12):1032-40.

68. Korsnes JS, Davis KL, Ariely R, Bell CF, Mitra D. Health care resource utilization and costs associated with nonfatal major adverse cardiovascular events. J Manag Care Spec Pharm. 2015;21(6):443-50. Available at: https:// www.jmcp.org/doi/10.18553/jmcp.2015.21.6.443.

69. Straka RJ, Liu LZ, Girase PS, DeLorenzo A, Chapman RH. Incremental cardiovascular costs and resource use associated with diabetes: an assessment of 29,863 patients in the U.S. managed-care setting. Cardiovasc Diabetol. 2009;8:53. 